\title{
Author Correction: Molecular subtypes of small cell lung cancer: a synthesis of human and mouse model data
}

Charles M. Rudin (D), John T. Poirier(D), Lauren Averett Byers, Caroline Dive (D), Afshin Dowlati, Julie George, John V. Heymach, Jane E. Johnson,

Jonathan M. Lehman, David MacPherson, Pierre P. Massion, John D. Minna, Trudy G. Oliver, Vito Quaranta, Julien Sage (D), Roman K. Thomas,

Christopher R. Vakoc and Adi F. Gazdar

Nature Reviews Cancer (2019) https://doi.org/10.1038/s41568-019-0133-9

Published online 29 March 2019

In the originally published article, some of the cell line names in the first column of Supplementary Table 1 were incorrect. The Supplementary Information to this Author Correction contains the correct version of Supplementary Table 1. This Supplementary Information is available in the online version of the Author Correction.

https://doi.org/10.1038/s41568-019-0164-2 I Published online 7 June 2019

Supplementary information

Supplementary information is available for this paper at https://doi.org/10.1038/

s41568-019-0164-2. 